Cahiers d'études italiennes

9| 2009

NOVECENTO... E DINTORNI

Images littéraires de la société contemporaine (4)

\title{
L'inquisition : au-delà de la religion. Regards croisés
}

Stefano Magni

\section{OpenEdition}

\section{Journals}

Édition électronique

URL : http://journals.openedition.org/cei/189

DOI : $10.4000 /$ cei. 189

ISSN : 2260-779X

\section{Éditeur}

UGA Éditions/Université Grenoble Alpes

\section{Édition imprimée}

Date de publication : 15 juillet 2009

Pagination : $57-67$

ISBN : 978-2-84310-145-8

ISSN : $1770-9571$

\section{Référence électronique}

Stefano Magni, «L'inquisition : au-delà de la religion. Regards croisés », Cahiers d'études italiennes [En ligne], 9 | 2009, mis en ligne le 15 janvier 2011, consulté le 27 mars 2021. URL : http:// journals.openedition.org/cei/189; DOI : https://doi.org/10.4000/cei.189 


\title{
L'INQUISITION : AU-DELÀ DE LA RELIGION. REGARDS CROISÉS
}

\author{
Stefano Magni \\ Université Stendhal - Grenoble 3
}

Si ces dernières années ont vu un regain d'intérêt pour la matière religieuse, la religion n'est pas un thème de choix pour la littérature italienne récente et ce n'est qu'occasionnellement que certaines œuvres contemporaines se sont intéressées au sentiment du religieux. Les romans que je me propose d'analyser ont abordé la matière religieuse par le biais du phénomène de l'inquisition. Il s'agit de La strega e il capitano, de Leonardo Sciascia, paru en 1986, de La chimera, de Sebastiano Vassalli, paru en 1990, et enfin de La strega e il capitano de Gino Songini, paru en 2000. Ces trois romans ont pour sujet des jeunes filles victimes des croyances et des préjugés du $\mathrm{XVII}^{\mathrm{e}}$ siècle et témoignent de trois positions idéologiques différentes. Afin de déterminer la place de la religion et du sentiment du religieux dans ces œuvres, je vais tout d'abord les étudier en tant que romans historiques ce qui me conduira à analyser leurs sources historiques et leur aspect fictionnel - dans le but de comprendre quel est le poids que l'Histoire de l'Église a dans la reconstruction historique et quel est le message chrétienreligieux, s'il y en a un, dans les figures de la fiction; je passerai ensuite à une analyse du regard que portent ces trois romans sur l'Inquisition et sur l'Église, pour aboutir enfin à des considérations plus générales sur le sens que peuvent avoir ces œuvres dans le contexte contemporain.

\section{L'Histoire, le réel et la fiction}

Ces trois romans historiques ont un même sujet et des histoires parallèles : la persécution de jeunes filles condamnées comme sorcières. Nous nous intéresserons tout d'abord au lien que ces textes entretiennent avec les 
données historiques de leurs récits. Dans les trois cas, les auteurs développent une histoire qui se situe à un moment historique précis : le XVII ${ }^{\mathrm{e}}$ siècle. Sciascia se réfère à un procès qui eut lieu en I616, Vassalli raconte les vicissitudes d'une fille née en 1590 et condamnée en I6IO, Songini situe son personnage un peu plus tard : son héroïne naît en 1676 et ne mourra qu'en I7I2. Cependant, bien que ces trois romans ont en commun le fait de se dérouler au cours du même siècle et de donner des dates précises, leur manière de traiter l'Histoire est différente.

Dans La strega e il capitano, Sciascia effectue une lecture herméneutique où les anecdotes et les faits de la fiction sont quasiment absents. Le texte se présente comme une exégèse des textes qui ont traité le sujet. Le narrateur déclare ses sources dès le début et part notamment de la lecture du chapitre xxxi des Promessi sposi, où Manzoni parle du protofisico Ludovico Sattala, l'homme de science qui s'opposa à la condamnation des "untori » - les semeurs de peste - mais qui, paradoxalement, fit condamner une jeune fille comme sorcière ${ }^{\mathrm{I}}$. Le narrateur a également consulté la source de Manzoni, à savoir la Storia di Milano de Pietro Verri, et il s'efforce d'en combler les vides. L'histoire dont il parle est documentée, car elle concerne un procès qui s'est déroulé à Milan en 1616 contre la servante Caterina Medici de' Brono, et relève donc clairement du plan de la réalité. Les actes du procès ont d'ailleurs été publiés en 1989 dans un ouvrage scientifique de Giuseppe Farinelli et Ermanno Paccagnini. Sciascia offre en permanence des dates, des observations textuelles et des repères chronologiques, comme par exemple : "A questo accenno, segue una lunga nota: che comincia alla pagina IS2 e si dislaga fino alla IS7, fittamente e quasi interamente occupando dunque ben sei pagine" (Sciascia, 1999, p. 13) ; et va jusqu'à donner une bibliographie pour qui veut aller plus loin : "Chi vuol saperne di più [...] sul noce di Benevento, può anche fermarsi alla Caccia alle streghe di Giuseppe Bonomo e al Paese di Cuccagna di Giuseppe Cocchiara" (Sciascia, 1999, p. 7I). Néanmoins sa reconstruction analyse les actes juridiques sans impliquer une réflexion historique sur la fonction de l'Église catholique dans le phénomène de la chasse aux sorcières.

Vassalli se réfere lui aussi à l'histoire d'une jeune fille dont il situe la mort en I6Io. Mais si on le compare au récit de Sciascia, ses sources (" certe carte ") sont dissimulées. Le fait de ne pas travailler sur des documents historiques identifiés porte l'écrivain à développer un récit qui est plutôt

I. A. Manzoni, 1985, cap. Xxxi, p. 56I : « [...] cooperò a far torturare, tanagliare e bruciare, come strega, una povera infelice sventurata, perché il suo padrone pativa dolori strani di stomaco, e un altro padrone di prima era stato fortemente innamorato di lei [...]." 
narratif qu'herméneutique. Son roman s'inspire directement des Promessi sposi (Magni, 2006, pp. 256-319) et offre une relecture du message éthique du roman fondateur de la littérature italienne. Comme Manzoni, Vassalli mêle les personnages historiques aux personnages fictifs. Son récit redécouvre les plaisirs de la narration, dépeint des caractères charmants et s'enrichit de la fluidité d'une diégèse fictionnelle.

Le narrateur donne parfois des dates qui pourraient créer des liens avec le plan du réel, mais ce ne sont que des références éphémères :

Francesca e Bartolo Nidasio comparvero davanti all'inquisizione il 28 di giugno, un lunedì: ed è certamente da annoverare tra le stranezze del processo di Antonia il fatto che i genitori della strega siano stati ascoltati così tardi, e dopo altri testimoni [...]. (Vassalli, 1990, p. 240)

Vassalli donne également la date du procès : le 20 août I6ıo (Vassalli, I990, p. 270 ${ }^{2}$. Il transcrit même, un extrait du manuscrit qu'il dit avoir trouvé (Vassalli, I990, pp. 286-287), mais il ne s'agit là que d'une citation ponctuelle, puisque le roman se consacre à une narration fictionnelle et ne jette pas de regard analytique sur le manuscrit ${ }^{3}$. Vassalli parle à peine des passages techniques du procès, car son intérêt tourne autour de l'histoire d'amour d'Antonia avec le "camminante » Gasparo et de la description de la vie de la campagne. Mais il nous donne néanmoins une image politique de l'Église catholique au travers des données historiques. Un exemple de cette tendance nous est donné par l'image du cardinal manzonien Federigo Borromeo qui est cité dans La chimera avec des traits contraires à ceux qui le caractérisent dans I promessi sposi : celui-ci est chez Vassalli un homme politique cynique et rusé, alors que dans le roman du XIX ${ }^{e}$ siècle il était un père affectueux.

Dans son roman qui est lui aussi une réécriture, Gino Songini utilise les matériaux historiques avec encore plus de parcimonie que Vassalli et cite très peu de passages historiques, ce qui fait de son roman le moins historique des trois. La date de la mort de la jeune fille nous est donnée dans la première page, mais la vie de la sorcière est ensuite reconstruite sans donner de repères chronologiques, et ne revient à une chronologie exacte que pour faire coïncider la fin de l'histoire. Il cite alors avec précision la

2. Il précise aussi l'heure : "Il 20 agosto alle quattro pomeridiane."

3. Comme l'attestent les études sur le sujet et comme nos recherches personnelles dans les archives l'ont démontré, les procès suivaient une structure rigide où l'inquisiteur cherchait, avec une série de passages obligés, de reconduire tout cas aux arguments universellement reconnus sur le sujet de la sorcellerie. Il s'agissait de faire avouer la rencontre charnelle avec le diable, le sacrifice d'enfants, les petits actes de sorcellerie pratiqués avec des herbes ou des cheveux, et ainsi de suite. Le "sabbat » étant un rite spécifique, l'accusation de participer aux sabbats apparaît seulement dans certaines cultures. 
date du procès de Giannina, le 26 octobre I7I2, un vendredi (Songini, 2000, p. 192). Ces documents historiques n'informent pas le lecteur sur la position de l'Église, position qui est en revanche décrite au travers d'images qui relèvent de la fiction. Les influences littéraires sont à peine voilées et le niveau de réécriture du roman de Songini va bien au-delà du texte de Sciascia dont il copie le titre. L'histoire s'inspire elle aussi des Promessi sposi, et se met de plus en rapport avec Vassalli, mais sa source d'inspiration est - me semble-t-il - Marco e Mattio plutôt que La Chimera.

Ainsi classés, ces romans montrent une progression qui va du plus historique au moins fictionnel et du plus narratif au moins documenté. Mais la perspective historique pourrait se révéler fallacieuse, car même le plus historique de ces textes, celui de Sciascia, dissimule un niveau de réécriture, car il reprend un roman du XIX ${ }^{\mathrm{e}}$ siècle qui avait déjà abordé le sujet : Caterina Medici di Brono. Novella Storica del secolo XVII de l'écrivain Achille Mauri, publié en I83i. La fidélité à l'Histoire nous permet de comprendre, somme toute, comment les auteurs ont comblé les espaces vides, en s'aidant de leur imagination et de leur idéologie, ce que je vais maintenant tenter de montrer au moyen d'une analyse du sens du religieux dans ces textes.

\section{Religion et laïcité}

Comme je l'ai déjà dit, les auteurs utilisent un matériau qui comporte un aspect laïque et un aspect religieux et il me semble intéressant d'étudier l'attention qu'ils portent à ce deuxième aspect. Mon premier exemple, Sciascia, laisse très peu d'espace au discours religieux. À cet égard, il faut aussi remarquer que la première source de Sciascia est la Storia di Milano, de Pietro Verri, intellectuel milanais surtout connu pour sa participation à la revue Il Caffé. Sous l'influence des idées réformistes des Lumières, Verri est porteur d'un message laïc, et dans ses écrits la religion est quasiment absente. Sciascia se situe idéalement dans un même courant en récupérant l'humanisme et la tradition illuministe et libérale de la pensée occidentale moderne. Dans le regard détaché de Sciascia sur la matière religieuse on peut donc déceler un symptôme de sa position idéologique, car pour Sciascia il s'agit d'une façon implicite de donner un ordre de valeurs. Les moments où la religion apparaît sont très rares. De plus, lorsqu'elle apparaît, la religion est présentée par le biais d'un autre sujet, d'une perspective différente. Une phrase au début de la narration nous permet d'illustrer cet aspect. Sciascia y définit la condamnation de la jeune fille comme un 
"fosco grappolo di atroce sofferenza, di feroce stupidità " (Sciascia, I999, p. I6) et, peu après, il pénètre dans le sujet du divin : à propos d'un personnage, Ludovico Melzi, fils de Luigi Melzi - l'homme qui déclanche la chasse à la sorcière avec son inexplicable mal au ventre - Sciascia écrit : "[...] in quel che Ludovico Melzi proclama aiuto divino ed è invece, semplicemente, l'aiuto di un cretino che non riconosce in sé il divino. Il divino dell'amore. Il divino della passione amorosa" (Sciascia, I999, p. I7). L'idée du divin est introduite par une réflexion sur la banalité de la souffrance des autres. On peut infliger à une jeune fille des peines indicibles, sans comprendre la souffrance qu'on provoque. On se réfere alors à la divinité pour ne pas regarder à l'intérieur de soi-même. Ludovico Melzi fait ainsi appel à l'aide divine parce qu'il est incapable d'analyser les âmes humaines. Le passage cité, est l'un des seuls cas où le narrateur se réfère directement au divin. Il écarte autrement le sujet en se concentrant sur l'analyse des textes et en faisant un effort pour reconstruire une histoire dont il n'a que des traces et à laquelle il tente de donner une logique.

Dans sa diégèse, Vassalli accueille en revanche les argumentations religieuses. Je me limite à remarquer qu'on peut signaler deux tendances intéressantes. La première concerne le dialogue que Vassalli instaure avec sa première source, I promessi sposi. Vassalli attaque le concept manzonien de "Provvidenza» et le renverse, dans une argumentation qui vise clairement I promessi sposi. Pour les orphelines du roman, la providence se trouve dans leur propre corps : "[...] la sola cosa che vi aiuterà ad affrontare il mondo è quell'affare che avete tra le gambe. Li c’è la Provvidenza, quella vera, l'unica che ci viene in aiuto anche quando il mondo intero ci è contro! " (Vassalli, I990, p. 34). La deuxième concerne les cultes et les phrases hérétiques dont on a par exemple des traces dans les mots d'Antonia. Pendant l'interrogatoire de l'Inquisition, la sorcière de Zardino, paraphrasant peut être les études de l'historien Carlo Ginzburg (Ginzburg, 1976), affirme qu'après la mort il n'y a rien : "[...] un gnente grande come il cielo, et in quel gnente le favole dei preti " (Vassalli, I990, p. 193). Elle nie, de plus, la nature divine de Jésus et la virginité de la Vierge et considère que la divinité correspond à la matière, aux quatre éléments ${ }^{4}$.

4. S. Vassalli, I990, p. 255 : "Il primo degli argomenti era l'inutilità dei preti, parassiti delle campagne e del mondo intero; il secondo, era la natura soltanto simbolica del Cristo ("Ce ne sono stati tanti Gesus Cristi, da che ce il mondo, et Gesucriste anco più assai"); il terzo, infine, era l'origine del peccato, ciò che la Chiesa chiama "peccato originale" e che secondo Antonia era la religione stessa. " C. Ginzburg, 1976, p. 7 : "[...] che credevù, che Gesù Cristo sia pasciuto della vergine Maria? non è possibile che l'habbia partorito et sia restata vergine" et " [...] l'aere è Dio [...] la terra è nostra madre; che vi maginate che sia Dio? Iddio non è altro che un può de fiato, et quello tanto che che l'hommo se immagina; 'l cielo, terra, mare, aere, abisso e inferno, tutto è Dio". 
Dans le roman de Gino Songini, la religion revêt un rôle important et il s'agit surtout des pratiques religieuses traditionnelles. L'auteur présente une vision courante de la religion qui arrive à travers les paroles du curé et dont on montre l'aspect le plus institutionnel et simpliste :

"Ancora più lontano" riprende don Lupo "[...] di là dai monti e di là dai mari vi sono popoli che non conoscono Dio né la sua legge. Adorano le pietre, le piante e gli animali, non sanno di avere un'anima e non rispettano i comandamenti che, prima che sulle tavole della legge, sono stati scolpiti nel cuore di ciascuno di noi." (Songini, 2000, p. 40)

Le curé est le représentant d'un monde paysan et restreint, lié à des valeurs anciennes et un peu folkloriques. Sa présence au sein de la communauté est celle qui nous arrive des images stéréotypées et figées de la campagne, pas nécessairement du XVII ${ }^{\mathrm{e}}$ siècle, éventuellement du siècle dernier. De même, les villageois reçoivent les préceptes religieux et en font leurs valeurs, en respectant les mœurs catholiques, comme il apparaît de certaines phrases : "E peccato desiderare la roba degli altri - aggiunse Elisabetta" (Songini, 2000, p. 75 ). Les mœurs de la religion catholique sont souvent au centre de l'intrigue.

Les figures des ecclésiastiques, en premier lieu de don Lupo, ont un poids considérable dans la narration, et le discours de la condamnation de la jeune Giannina mêle les aspects sociaux aux aspects religieux, comme on le voit déjà lors de la découverte de sa maternité illégitime :

Vostra figlia non mi ha voluto ascoltare, Antonio! La parola del sacerdote che esercita il suo ministero è quella della Chiesa, è la parola di Dio che si rivela agli uomini. Giannina invece ha voluto dare ascolto alla voce del Maligno. Un figlio è una benedizione del Signore, ma questo è soltanto il frutto del peccato. E il peccato è maledetto da Dio. "L'inferno ha allargato la sua anima", come ha detto Isaia. Ma c'è un altro aspetto, ancora più grave: lo scandalo! Vostra figlia ha dato scandalo! E per chi dà scandalo non ci può essere pietà. Meglio per lui legarsi una macina da mulino al collo e gettarsi nella profondità degli abissi. (Songini, 2000, p. I45)

Les deux argumentations - sociale et religieuse - procèdent de pair. Le petit monde ancien qui sort de cette description est donc fortement catholique. Analysons maintenant comment ces différentes positions peuvent nous donner la position idéologique des auteurs et expliquer en même temps les visées de l'œuvre littéraire. 


\section{Visées de l'argumentation religieuse}

On peut constater que la position de Songini donne beaucoup de place à la voix morale de la religion et de l'Église, que dans le livre de Sciascia les figures des curés sont absolument secondaires et que la position de Vassalli est intermédiaire (les figures des curés sont nombreuses, mais leur explication est complexe). Vassalli montre un monde religieux séparé du monde séculaire. De son discours religieux on peut retenir le fait qu'il accuse l'Église catholique d'avoir contribué à jeter dans l'oubli une grande richesse culturelle païenne, surtout après le Concile de Trente (terminé en 1564). Au-delà donc des figures plus ou moins humaines, plus ou moins lâches ou plus ou moins bureaucrates, le roman de Vassalli propose un débat très intéressant sur orthodoxie et hérésie, qui va aussi au-delà de l'histoire personnelle de la sorcière. L'élimination des sorcières était d'ailleurs l'un des aspects de l'élimination des hérésies. Le roman de Vassalli pose donc aussi la question des luttes politiques et religieuses pour l'affirmation des religions les unes sur les autres. Vassalli questionne ainsi le sentiment du religieux, mais critique surtout nos structures civiles et sociales. En critiquant l'Église catholique en tant que structure du pouvoir, implicitement, peut être, Vassalli critique aussi la pensée de l'un des plus importants penseurs de la période, Thomas Hobbes (I588-I679). Selon Hobbes, l'homme était naturellement en guerre avec les autres hommes (Homo homini lupus) et seulement l'ordre d'un gouvernement (ou mieux le gouvernement d'un seul homme) pouvait éliminer la pluralité des positions et permettre la vie en commun. À partir du moment où, dans le roman, c'est toujours l'institution religieuse ou laïque qui est un loup pour l'homme, on met en discussion les institutions mêmes et on porte une attaque nihiliste, voire postmoderne, aux institutions du monde occidental. Dans son message, on peut tout de même trouver une confrontation personnelle avec le sens du religieux. Dans la dernière partie de son livre, Il nulla - Congedo, Vassalli s'exprime sur le religieux avec une phrase très forte qui déclare ouvertement une "foi " dans l'athéisme, lorsqu'il parle de : "Colui che conosce il prima e il dopo e le ragioni del tutto e però purtroppo non può dircele per quest'unico motivo, cosi futile!: che non esiste " (Vassalli, I990, p. 255). La violence idéologique de cette conclusion enlève toute illusion de rédemption humaine, comme si des siècles entiers avaient été gaspillés dans l'illusion religieuse. Pour l'auteur, ce n'est pas la foi qui est garante des valeurs éternelles, mais c'est la valeur de la mémoire qui donne espoir à l'humanité. À la foi en Dieu, Vassalli 
oppose une foi dans la transmission du savoir, et en particulier " un culte " des livres. Mais en même temps, le fait de conclure son roman avec une référence à la divinité indique aussi un intérêt, de la part de l'auteur, pour la spiritualité. On peut lire dans cette conclusion une ambition, un élan vers la spiritualité, ou tout simplement un besoin de confrontation avec le thème du religieux.

Cette confrontation semble absente du livre de Sciascia. Sciascia néglige, comme je l'ai dit, les figures des curés et reconduit l'histoire de Caterina à la situation sociologique de l'époque. Il se livre à une écriture scientifique qui enlève pathos et participation émotive à la description des personnages, et analyse attentivement les rapports entre la servante et son maître et les dynamiques sociales qui réglaient la vie des familles nobles. De même, son regard sur la sorcellerie est inspiré par des réflexions sociales et ne tient pas compte de l'aspect religieux. Le fait de mettre en rapport, comme le dit le titre, la jeune sorcière avec le capitaine de justice, est ainsi un moyen d'écarter le problème du religieux et de reconduire la sorcellerie à l'ordre de la magie. De cette façon, Sciascia évite de parler de l'orthodoxie religieuse et se concentre plutôt sur la position des hommes de sciences, les médecins, par exemple - dont il transcrit les témoignages - en critiquant ainsi fortement la tradition du logos et de la techné occidentale. Au $\mathrm{XVII}^{\mathrm{e}}$ et XVIII ${ }^{\mathrm{e}}$ siècle, la pensée scientifique occidentale se constitue en tant que telle et Sciascia, avec sa critique, attribue à la science de l'époque des croyances et des préjugés a-scientifiques. Il récupère la tradition scientiste, mais en même temps il la critique, pour l'approximation de ses méthodes. De même que, parlant de Vassalli, j'ai cité Hobbes, parlant de Sciascia, je pourrais évoquer l'héritage de Locke (I632-I704), de Hume (I7II-I776) et de Voltaire (I694-I778). La religion, dans l'argumentation de Sciascia, est la négation de la rationalité. Il me semble que c'est à travers cette optique qu'on peut comprendre le sens de cet ouvrage. Et, sans négliger les figures des curés présentes dans les policiers de l'écrivain sicilien, la description de Don Antonio tirée du roman Candido, ovvero un sogno fatto in Sicilia, me semble fort éclairante pour compléter la vision sur la religion. Don Antonio se défroque et adhère au Parti Communiste (Sciascia écrit ce roman en 1977 , lors de sa rupture avec le Parti communiste italien). Dans la dernière image, très réussie, Don Antonio, à Paris, rend hommage à la statue de Voltaire :

Dal quai, imboccarono rue de Seine. Davanti alla statua di Voltaire don Antonio si fermò, si afferrò al palo della segnaletica, chinò la testa. Pareva si fosse messo a pregare. - Questo è il nostro padre - gridò poi - questo è il nostro vero padre. (Sciascia, I990, p. 460) 
Si Sciascia et Vassalli se situent du côté d'une pensée lä̈que, Songini finit son texte en remémorant un passage de la Bible : le comandant des gardes, qui vient de notifier aux parents de la sorcière l'exécution de la jeune fille, n'a pas le courage de leur dire que la loi les charge aussi des dépenses de l'exécution même. Il décide alors de couvrir lui-même les frais en rappelant un épisode de la Bible :

Trenta lire sono pronte. A breve verserò la differenza, anche perché ho un credito di dieci lire con il servitore curiale. E non ha importanza se non mi rimarrà un centesimo. Del resto nemmeno i capi dei sacerdoti vollero mettere in cassa le trenta monete d'argento riportate da Giuda. Nemmeno loro vollero trattenere denaro giudicato "prezzo del sangue". (Songini, 2000, p. 203)

De plus, on peut remarquer que Songini, dans sa préface, rappelle le pardon que le Pape a demandé pour les victimes de la persécution de l'Église catholique, observation dans laquelle on perçoit, une fois de plus, une perspective catholique :

Il I2 marzo dell'anno giubilare 2000, il Papa ha chiesto perdono per tutte le vittime (quante, mio Dio!) della persecuzione clericale. La chiesa martire delle catacombe, rinnegando se stessa, è divenuta nei secoli persecutrice e carnefice di tanti innocenti. È stato un gran giorno, il I2 marzo 2000. (Songini, 2000, p. 22)

Dans cette phrase, on remarque l'argumentation éthique du narrateur qui met en regard le passé des martyres chrétiens et la « Némésis » de la persécution de l'Inquisition.

Ces passages permettent aussi d'éclairer l'importance donnée à la figure du curé dans le roman, et le fait que l'auteur semble parfois observer d'un regard nostalgique la simplicité du monde paysan et catholique d'autrefois. Songini intègre son curé à la communauté, et le degré ironique est parfois subtil. Certes, le nom du curé même, Lupo, est déjà révélateur de son rôle diégétique, mais l'on a parfois l'impression que le narrateur est lui aussi charmé par l'authenticité et la simplicité de son monde ancien.

Songini part donc de l'idée de réécrire le roman de Sciascia, et il se réfère aussi clairement à Vassalli, mais au final, il nous livre un message qui differe des deux autres auteurs. Dans son roman, une jeune fille est victime de l'inquisition pour le seul fait que, sans être mariée, elle a eu un enfant. Mais sa critique ne concerne pas seulement les structures sociales qui ont mené à cette fin, mais aussi les mœurs catholiques qui, comme je viens de le dire, d'un côté sont symboles d'amour et pitié et de l'autre ont su atteindre un tel degré d'intolérance. 
Pour conclure, on pourrait dire que dans le roman de Vassalli, on trouve aussi une critique, mais plus voilée, de la contradiction du monde de la chrétienté, l'image qui le montre le plus étant à mon avis la description du trajet qui mène Antonia à l'échafaud et qui rappelle le Calvaire subi par Jésus. Fils de la tradition illuministe et laïque de la pensée occidentale moderne, Sciascia achève en revanche son roman avec une référence au concept laïque de justice : "E cosi - assicurò il boia - giustizia fu fatta" (Sciascia, I999, p. 80). Les principes de la morale priment sur les valeurs de la foi et sur la connaissance de Dieu. Parmi ces différences, on peut néanmoins remarquer que les trois auteurs ont choisi d'observer la religion lorsqu'elle se présente comme persécutrice. Des observations sur la période peuvent enrichir mes analyses. Sciascia publie son roman en 1986, l'année où Primo Levi fait sortir I sommersi e $i$ salvati, sa dernière réflexion sur les camps de concentration, où l'appartenance à une religion, le judaïsme, est la cause de la persécution. Entre temps, les Balkans ont connu, pendant la guerre dans l'ex-Yougoslavie, la persécution des musulmans, un autre exemple d'une chasse aux sorcières, où encore une fois le fait d'appartenir à une religion différente est la raison de la persécution même. Le choix de montrer la religion comme persécutrice peut se prêter à plusieurs interprétations et, comme j'ai tenté de le dire, peut être reconduit à plusieurs choix idéologiques car, dans ces livres, le sens du religieux se mêle avec l'histoire de notre civilisation. Ces auteurs ont donc montré trois approches différentes qui nous obligent à nous confronter avec notre culture, et ouvrent un débat aussi vaste qu'intéressant.

\section{Bibliographie}

Farinelli G. e Paccagnini E., Processo per stregoneria a Caterina de Medici, Milano, Rusconi, 1989.

Ginzburg C., Il formaggio e i vermi: il cosmo di un mugnaio del 'soo, Torino, Einaudi, 1976.

Magni S., Le détournement des genres dans la production narrative postmoderne en Italie, thèse de doctorat sous la direction de $\mathrm{M}^{\mathrm{me}}$ le professeur Marina Fratnik, soutenue à l'Université Paris 8 Saint-Denis le $30 / 10 / 2006$.

Manzoni A., I promessi sposi (I823-1840), dans Tutte le opere, Milano, Mondadori, 1985.

Mauri A., Caterina Medici di Brono. Novella Storica del secolo XVII, Milano, Luigi Nervetti, I83I. 
Sciascia L., La strega e il capitano, Milano, Adelphi, 1999 [1986].

-, Candido, ovvero un sogno fatto in Sicilia (1977), dans Opere, Milano, Bompiani, I990, vol. II.

Songini G., La strega e il capitano, Milano, Greco \& Greco, 2000.

Vassalli S., La chimera, Torino, Einaudi, 1990.

—, Marco e Mattio, Torino, Einaudi, 1992. 
